\title{
Solução Iterativa dos Sistemas Lineares do Método de Pontos Interiores
}

\author{
C.T.L.S. GHIDINI ${ }^{1 *}$, A.R.L. OLIVEIRA ${ }^{2}$ e M. SILVA $^{3}$,
}

Recebido em 28 novembro, 2013 / Aceito em 27 agosto, 2014

\begin{abstract}
RESUMO. Nesse trabalho, consideramos o método preditor-corretor, que é uma das variantes mais importante do método de pontos interiores devido à sua eficiência e convergência rápida. No método preditorcorretor, é preciso resolver dois sistemas lineares a cada iteração para determinar a direção preditoracorretora. A resolução desses sistemas é o passo que requer mais tempo de processamento, devendo assim ser realizada de maneira eficiente. Para obter a solução dos sistemas lineares do método preditor-corretor consideramos dois métodos iterativos de Krylov: MINRES e método dos gradientes conjugados. Para que estes métodos convirjam mais rapidamente um pré-condicionador especialmente desenvolvido para os sistemas lineares oriundos dos métodos de pontos interiores é usado. Experimentos computacionais em um conjunto variado de problemas de programação linear foram realizados com o intuito de analisar a eficiência e robustez dos métodos de solução dos sistemas.
\end{abstract}

Palavras-chave: métodos de pontos interiores, sistemas lineares, métodos iterativos.

\section{INTRODUÇÃO}

O trabalho de Karmarkar em 1984 [6] revolucionou a área de programação linear por apresentar um algoritmo com complexidade polinomial e excelente desempenho quando aplicado em problemas lineares de grande porte. A partir daí, foi desenvolvido uma nova linha de pesquisa: os métodos de pontos interiores. Esses métodos buscam a solução ótima de um problema de programação linear, percorrendo o interior da região de factibilidade, determinada pelas restrições do problema. Eles podem ser classificados em primal, dual e primal-dual ou ainda por métodos afim escala, método de redução de potencial e métodos de trajetória central.

Neste trabalho, estamos considerando o método preditor-corretor, que é um método do tipo primal-dual de trajetória central. O passo mais importante do método preditor-corretor consiste

\footnotetext{
*Autor correspondente: Carla Ghidini

${ }^{1}$ FCA - Faculdade de Ciências Aplicadas, UNICAMP - Universidade Estadual de Campinas, 13484-350 Limeira, SP, Brasil. E-mail: carla.ghidini@fca.unicamp.br

2 Departamento de Matemática Aplicada, IMECC - Instituto de Matemática, Estatística e Computação Científica, UNICAMP - Universidade Estadual de Campinas, 13083-859 Campinas, SP, Brasil. E-mail: aurelio@ ime.unicamp.br ${ }^{3}$ Departamento de Matemática Aplicada, IMECC - Instituto de Matemática, Estatística e Computação Científica, UNICAMP - Universidade Estadual de Campinas, 13083-859 Campinas, SP, Brasil. E-mail: marilene @ ime.unicamp.br
} 
em encontrar as direções preditora (direção afim escala) e corretora. Para isso, alguns sistemas de equações lineares devem ser resolvidos a cada iteração, o que requer grande esforço computacional.

Uma forma de diminuir a complexidade computacional consiste em reduzir esses sistemas a sistemas de equações normais ou sistemas aumentados e depois aplicar algum método iterativo para resolvê-los.

Para resolver os sistemas de equações normais e aumentado foi feita uma implementação cuidadosa do método MINRES com reortogonalização [16]. Esta implementação foi comparada com uma implementação do método dos gradientes conjugados (GC) já existente [15]. Ambos métodos utilizam o subespaço de Krylov como subespaço de busca para encontrar uma solução aproximada do sistema, o que é uma estratégia vantajosa, uma vez que esse subespaço pode ser muito menor que o subespaço de busca completo.

Para que os métodos iterativos tenham um melhor desempenho é realizado o pré-condicionamento da matriz de restrições dos sistemas utilizando o pré-condicionador separador. Além disso, com o intuito de resolver um número maior de problemas e de forma mais eficiente é considerada uma abordagem que utiliza, inicialmente, o GC e depois de um certo número de iterações, se o critério pré-estabelecido para troca for satisfeito, o MINRES passa a ser utilizado. Mais detalhes sobre essa técnica são fornecidos na Seção 5.

\section{MÉTODO DE PONTOS INTERIORES - PRIMAL DUAL}

Considere o problema de programação linear na forma padrão:

$$
\begin{array}{ll}
\text { Min } & c^{t} x \\
\text { s.a. } & A x=b \\
& x \geq 0,
\end{array}
$$

em que $A \in \mathbb{R}^{m \times n}, \operatorname{posto}(A)=m, x \in \mathbb{R}^{n}, c \in \mathbb{R}^{n}$ e $b \in \mathbb{R}^{m}$. Associado ao problema (2.1), denominado de problema primal, temos o seguinte problema dual:

$$
\begin{array}{ll}
\operatorname{Max} & b^{t} y \\
\text { s.a. } & A^{t} y+z=c \\
& z \geq 0,
\end{array}
$$

em que $y \in \mathbb{R}^{m}$ é o vetor de variáveis duais livres e $z \in \mathbb{R}^{n}$ de variáveis de folga.

As condições de otimalidade de primeira ordem (Karush-Kuhn-Tucker) de (2.1) e (2.2) são dadas por [18]:

$$
\left\{\begin{array}{l}
A x-b=0 \\
A^{t} y+z-c=0 \\
X Z e=0 \\
(x, z) \geq 0
\end{array}\right.
$$

sendo $X=\operatorname{diag}(x), Z=\operatorname{diag}(z)$ e $e \in \mathbb{R}^{n}$, tal que $e=(1,1, \ldots, 1)^{t}$. 
Os métodos de pontos interiores do tipo primal-dual consistem em aplicar o método de Newton às condições de otimalidade (2.3), partindo de um ponto interior e mantendo interior a cada iteração. A direção afim escala (direção de Newton) é obtida resolvendo o seguinte sistema:

$$
\left[\begin{array}{ccc}
A & 0 & 0 \\
0 & A^{t} & I \\
Z & 0 & X
\end{array}\right]\left[\begin{array}{c}
\hat{d} x \\
\hat{d} y \\
\hat{d} z
\end{array}\right]=\left[\begin{array}{c}
r_{p} \\
r_{d} \\
r_{a}
\end{array}\right]
$$

em que $r_{p}=b-A x, r_{d}=c-A^{t} y-z, r_{a}=-X Z e$. Eliminando a variável $\hat{d} z$, obtemos o sistema aumentado:

$$
\left[\begin{array}{cc}
-D^{-1} & A^{t} \\
A & 0
\end{array}\right]\left[\begin{array}{c}
\hat{d} x \\
\hat{d} y
\end{array}\right]=\left[\begin{array}{c}
r_{d}-X^{-1} r_{a} \\
r_{p}
\end{array}\right]
$$

em que $D=X Z^{-1}$. A forma mais usada para resolver o sistema (2.4) consiste em reduzi-lo, por meio da eliminação da variável $\hat{d} x$, a um sistema de equações normais $A D^{-1} A^{t}$ :

$$
\left(A D A^{t}\right) \hat{d} y=A D\left(r_{d}-X^{-1} r_{a}\right)+r_{p} .
$$

Em geral, não é possível realizar um passo completo ao longo da direção de Newton, visto que $(x, z) \geq 0$ deve ser satisfeita. Dessa forma, o novo ponto é dado por:

$$
\left(x^{k+1}, y^{k+1}, z^{k+1}\right)=\left(x^{k}, y^{k}, z^{k}\right)+\hat{\alpha}\left(\hat{d} x^{k}, \hat{d} y^{k}, \hat{d} z^{k}\right),
$$

em que $\hat{\alpha}=\left(\hat{\alpha}_{p}, \hat{\alpha}_{d}, \hat{\alpha}_{d}\right)^{t}$. Os passos $\hat{\alpha}_{p}$ e $\hat{\alpha}_{d}$ são os passos primal e dual que preservam a não negatividade das variáveis $x$ e $z$, respectivamente. Esses valores são determinados da seguinte forma:

$$
\begin{aligned}
& \hat{\alpha}_{p}=\min \left\{1, \tau \min _{i}\left(-\frac{x_{i}^{k}}{\hat{d} x_{i}^{k}} \mid \hat{d} x_{i}^{k}<0\right)\right\}, \\
& \hat{\alpha}_{d}=\min \left\{1, \tau \min _{i}\left(-\frac{z_{i}^{k}}{\hat{d} z_{i}^{k}} \mid \hat{d} z_{i}^{k}<0\right)\right\},
\end{aligned}
$$

em que $\tau \in(0,1)$.

\subsection{Método Preditor-Corretor}

O método preditor-corretor desenvolvido por Mehrotra [9] consiste em utilizar uma direção composta por três componentes: direção afim escala, direção de centragem e direção de correção não linear [12]. Fazendo a correção não linear e introduzindo a perturbação para centragem, temos o sistema:

$$
\begin{aligned}
& A \bar{d} x=0 \\
& A^{t} \bar{d} y+\bar{d} z=0 \\
& Z \bar{d} x+X \bar{d} z=\mu e-\hat{D}_{x} \hat{D}_{z} e
\end{aligned}
$$

em que $\mu=\left(\frac{\hat{\gamma}}{\gamma}\right)^{p}\left(\frac{\hat{\gamma}}{n}\right), \gamma=\left(x^{k}\right)^{t} z^{k}$ e $\hat{\gamma}=\left(x^{k}+\hat{\alpha_{p}} \hat{d} x\right)^{t}\left(z^{k}+\hat{\alpha_{d}} \hat{d} z\right)$. 
Mehrotra em [9] sugere $p=2$ ou $p=3$. Além disso, ao tomar $\hat{\alpha}_{p}=\hat{\alpha}_{d}=1$, obtemos um ponto primal e dual factível. Assim, $r_{p}=r_{d}=0$ e $r_{a}$ na restrição de complementariedade é dado por $r_{a}=\hat{D}_{x} \hat{D}_{z} e$, em que $\hat{D}_{x}=\operatorname{diag}(\hat{d} x)$ e $\hat{D} z=\operatorname{diag}(\hat{d} z)$.

A direção preditora-corretora é dada por: $d=\hat{d}+\bar{d}$ e determinada resolvendo o sistema:

$$
\begin{aligned}
& A d x=r_{p} \\
& A^{t} d y+d z=r_{d} \\
& Z d x+X d z=r_{s}
\end{aligned}
$$

em que $r_{s}=r_{a}+\mu e-\hat{D}_{x} \hat{D}_{z} e$.

\subsection{Pré-condicionador Separador}

Para uma convergência mais rápida dos métodos iterativos o pré-condicionamento da matriz de restrições do sistema linear se faz necessário. O pré-condicionador separador [14] é específico para os sistemas lineares oriundos dos métodos de pontos interiores. Quando aplicado ao método dos gradientes conjugados evita o cálculo da matriz de equações normais e sua fatoração. Apresenta resultados muito bons próximos a uma solução do problema, mas não é eficiente nas primeiras iterações. Este pré-condicionador é calculado como segue: Considere a matriz de equações normais em (2.5). Tome $A=\left[\begin{array}{ll}B & N\end{array}\right] P$, em que $P \in \mathbb{R}^{n \times n}$ é uma matriz de permutação tal que $B \in \mathbb{R}^{m \times m}$ é não singular, então

$$
\begin{aligned}
A D A^{t} & =[B \quad N] P D P^{t}\left[\begin{array}{c}
B^{t} \\
N^{t}
\end{array}\right] \\
& =\left[\begin{array}{ll}
B & N
\end{array}\right]\left[\begin{array}{cc}
D_{B} & 0 \\
0 & D_{N}
\end{array}\right]\left[\begin{array}{c}
B^{t} \\
N^{t}
\end{array}\right] \\
& =B D_{B} B^{t}+N D_{N} N^{t} .
\end{aligned}
$$

O pré-condicionador é dado por: $D_{B}^{-\frac{1}{2}} B^{-1}$ e a matriz pré-condicionada $M$ é como segue:

$$
M=D_{B}^{-\frac{1}{2}} B^{-1}\left(A D A^{t}\right) B^{-t} D_{B}^{-\frac{1}{2}}=I_{m}+G G^{t},
$$

em que

$$
G=D_{B}^{-\frac{1}{2}} B^{-1} N D_{N}^{\frac{1}{2}}
$$

Note que próximo a uma solução pelo menos $n-m$ elementos em $D^{-1}$ são grandes. Dessa forma, uma escolha adequada das colunas de $B$ faz com que os elementos em $D_{B}^{-1 / 2}$ e $D_{N}$ sejam muito pequenos nesta situação. Neste caso, $G$ aproxima-se da matriz nula, $M$ se aproxima da matriz identidade e ambos, o menor e o maior autovalor de $M$, se aproximam do valor 1 , assim como o número de condição $\kappa_{2}(M)^{4}$.

\footnotetext{
${ }^{4} \mathrm{O}$ número de condição de uma matriz $M$ é definido por: $\kappa_{2}(M)=\|M\|_{2}\left\|M^{-1}\right\|_{2}$.
} 


\section{MÉTODOS DO SUBESPAÇO DE KRYLOV}

Considere o sistema linear:

$$
A x=b,
$$

em que $b$ é um vetor do $\mathbb{R}^{m}$ e $A$ é uma matriz $m \times n$. Sejam $\mathcal{K}$ e $\mathcal{L}$ dois subespaços do $\mathbb{R}^{n}$ de dimensão $n$. Uma técnica de projeção consiste em encontrar em $\mathcal{K}$ uma solução aproximada $\hat{x}$ para (3.1), de forma que o resíduo $b-A x$ seja ortogonal à $\mathcal{L}$, em que $\mathcal{L}$ denota o espaço de restrições.

Métodos do subespaço de Krylov são baseados em processos de projeção em subespaços de Krylov. O subespaço de Krylov de ordem $m$ associado à $A$ e $b$ é o subespaço gerado pelos vetores da sequência de Krylov: $\mathcal{K}^{m}(A, b)=\operatorname{span}\left\{b, A b, A^{2} b, \ldots, A^{m-1} b\right\}$. Nos métodos de Krylov, $\mathcal{K}^{m}$ é dado por: $\mathcal{K}^{m}\left(A, r^{0}\right)=\operatorname{span}\left\{r^{0}, A r^{0}, A^{2} r^{0}, \ldots, A^{m-1} r^{0}\right\}$, em que $r^{0}=b-A x^{0}$ é o resíduo da solução inicial $x^{0}$.

As diferentes versões dos métodos do subespaço de Krylov surgem a partir de diferentes escolhas do subespaço $\mathcal{L}^{m}$ e das formas pelas quais o sistema é pré-condicionado. Os métodos GC e MINRES foram originados escolhendo $\mathcal{L}^{m}=A \mathcal{K}^{m}$.

\subsection{Método de Arnoldi}

O método de Arnoldi é um método de projeção ortogonal [10] utilizado para construir uma base ortonormal para o subespaço de $\operatorname{Krylov} \mathcal{K}^{m}(A, b)$. Este procedimento começa com $v_{1}=\frac{r^{0}}{\left\|r^{0}\right\|_{2}}$. Em seguida, calcula-se $A v_{1}$ e a partir dele constrói-se um vetor ortogonal a $v_{1}$. Normaliza-se este vetor e obtém-se $v_{2}$. Dessa forma, se $v_{1}, \ldots, v_{j}$ for uma base ortogonal para $\mathcal{K}^{j}\left(A, r^{0}\right)$, então para encontrar $v_{j+1}$ basta calcular $t=A v_{j}$ e ortonormalizá-lo com respeito a $v_{1}, \ldots, v_{j}$. Isto produz um método para a criação de uma base ortonormal para $\mathcal{K}^{j+1}\left(A, r_{0}\right)$. Os vetores $v_{1}, \ldots, v_{j+1}$ formam uma base para $\mathcal{K}^{j+1}\left(A, r_{0}\right)$, a menos que $t$ seja igual a zero. A ortogonalização gera a relação expressa em termos de $v_{j}: A V_{m-1}=V_{m} H_{m, m-1}$, em que $V_{m}$ denota a matriz com colunas $v_{1}$ até $v_{m}$. A matriz $H_{m, m-1}$ é uma matriz Hessenberg superior de ordem $m \times m-1$, em que os elementos $h_{i, j}$ são definidos pelo método de Arnoldi.

A princípio o processo de ortogonalização pode ser realizado de diferentes formas, mas normalmente a abordagem mais usada é o processo de Gram-Schimidt [4].

\subsection{Método de Lanczos}

Assim como o método de Arnoldi, o método de Lanczos [8] é utilizado para gerar uma base ortonormal para um subespaço de Krylov associado à $A$ e $b$. Porém, em Lanczos $A$ deve ser uma matriz simétrica. Note que se $A$ é simétrica, então $H_{m-1, m-1}=V_{m-1}^{t} A V_{m-1}$ é uma matriz tridiagonal. Assim, durante o processo de ortogonalização de $A$, vários termos $h_{i j}$ se anulam, o que reduz $H$ a uma matriz tridiagonal simétrica e faz com que o custo computacional associado à ortogonalização e ao armazenamento sejam reduzidos, em comparação ao método de Arnoldi. 
O processo de Lanczos aproxima uma matriz simétrica $A$ em uma matriz simétrica tridiagonal $T_{k+1, k}{ }^{5}$ com uma linha adicional na parte inferior.

Se definirmos $T_{k}$ como sendo as primeiras $k$ linhas de $T_{k+1, k}$, então $T_{k}$ é uma matriz quadrada e simétrica. Dessa forma:

$$
T_{k+1, k}=\left[\begin{array}{c}
T_{k} \\
\beta_{k+1} e_{k}^{t}
\end{array}\right] \quad \text { e } \quad T_{k}=\left[\begin{array}{cc}
T_{k-1} & \beta_{k} e_{k-1} \\
\beta_{k} e_{k-1}^{t} & \alpha_{k}
\end{array}\right],
$$

em que $e_{k}^{t}$ representa o vetor canônico. O processo Lanczos determina o vetor $v_{k+1}$ tomando como vetor inicial $v_{0}=0$ e $\beta_{1} v_{1}=b$, em que $\beta_{1}$ serve para normalizar $v_{1}: \beta_{k+1} v_{k+1}=$ $p_{k}-\alpha_{k} v_{k}-\beta_{k} v_{k-1}$, em que $\alpha_{k}=v_{k}^{t} p_{k}$ e $p_{k}=A v_{k}$. O valor $\beta_{k+1}$ é usado para normalizar $v_{k+1}$. Na forma matricial,

$$
A V_{k}=V_{k+1} T_{k+1, k}, \text { em que } V_{k}=\left[v_{1}|\cdots| v_{k}\right]
$$

Em aritmética exata, as colunas de $V_{k}$ são vetores colunas ortonormais. O processo termina quando $\beta_{k+1}=0(k \leq n)$, então obtemos $A V_{k}=V_{k} T_{k}$.

Seja $A \in \mathbb{R}^{n \times n}$ uma matriz simétrica e $v_{1} \in \mathbb{R}^{n}$ um vetor unitário. Então, a partir do processo de Lanczos, obtemos a relação garantida pelo Teorema $9.11 \mathrm{em} \mathrm{[4]:} A V_{k}=V_{k} T_{k}+r_{k} e_{k}^{t}$.

\subsection{Método dos Gradientes Conjugados}

O método dos Gradientes Conjugados (GC) resolve sistemas de equações lineares, cuja matriz dos coeficientes é simétrica e definida positiva. Este método é baseado em Lanczos, a partir do qual é obtida uma base ortogonal de vetores para o subespaço de $\operatorname{Krylov} \mathcal{K}^{k}\left(A, r_{0}\right)$. Além disso, o método GC é um método fundamentado na abordagem de Ritz-Galerkin. Na condição de RitzGalerkin, o novo resíduo $b-A x_{k+1}$ deve ser ortogonal ao subespaço gerado por $v_{1}, \ldots, v_{k}$, ou seja, $V_{k}^{t}\left(b-A x_{k}\right)=0$.

Sejam $b=\beta_{1} v_{1}$ e $e_{1}$ o primeiro vetor canônico unitário em $\mathbb{R}^{k}$, segue que

$$
V_{k}^{t} b=V_{k}^{t}\left(\beta_{1} v_{1}\right)=\beta_{1}=\beta_{1} e_{1}
$$

visto que os vetores $v_{1}, \ldots, v_{k}$ formam uma base ortonormal para o subespaço de Krylov $\mathcal{K}^{k}(A, b)$. Procuramos por uma solução aproximada $x_{k}$ para o sistema $A x=b$ tal que $x_{k} \in$ $\mathcal{K}^{k}(A, b)$. Dessa forma, podemos escrever $x_{k}$ como uma combinação dos vetores da base de

$$
{ }^{5} T_{k+1, k}=\left[\begin{array}{ccccc}
\alpha_{1} & \beta_{2} & & & \\
\beta_{2} & \alpha_{2} & \beta_{3} & & \\
& \beta_{3} & \alpha_{3} & \ddots & \\
& & \ddots & \ddots & \beta_{k} \\
& & & \beta_{k} & \alpha_{k} \\
& & & & \beta_{k+1}
\end{array}\right] \text {, em que } \alpha_{j}=h_{i j}, \beta_{j}=h_{j-1, j} .
$$


$\mathcal{K}^{k}(A, b)$, ou seja, $x_{k}=V_{k} y$. Portanto, a condição $V_{k}^{t}\left(b-A x_{k}\right)=0$ pode ser reescrita como: $V_{k}^{t} A V_{k} y=\beta_{1} e_{1}$.

Claramente, precisamos construir a matriz $V_{k}^{t} A V_{k}$. Entretanto, da relação $A V_{k}=V_{k} T_{k}$, obtida a partir do processo de ortogonalização de Lanczos, segue que $V_{k}^{t} A V_{k}=T_{k}$. Assim, obtemos $x_{k}$, tal que $r_{k} \perp \mathcal{K}^{k}\left(A, r_{0}\right)$, resolvendo o seguinte sistema: $T_{k} y=\beta_{1} e_{1}$.

Em cada iteração do método dos Gradientes Conjugados, de acordo com [4, Seção 9.31], podemos resolver o sistema $T_{k} y_{k}=\beta_{1} e_{1}$ aplicando a fatoração de Cholesky na matriz $T_{k}$ do processo de Lanczos.

\subsection{Método MINRES}

O método MINRES resolve sistemas lineares simétricos indefinidos. Métodos baseados na norma mínima residual, como o MINRES, determinam a solução aproximada $x_{k} \in \mathcal{K}^{k}\left(A, r_{0}\right)$ do sistema $A x=b$ de tal forma que a norma Euclidiana $\left\|b-A x_{k}\right\|_{2}$ seja mínima sobre $\mathcal{K}^{k}\left(A, r_{0}\right)$.

Nas Seções 3.1 e 3.2, apresentamos dois processos de construção de uma base ortogonal para um subespaço de Krylov $\mathcal{K}\left(A, r^{0}\right)$ que produziram a relação $A V_{k}=V_{k+1} H_{k+1, k}$, em que $V_{k}$ é uma matriz cujas colunas $v_{1}, \ldots, v_{k}$ formam a base do subespaço $\mathcal{K}^{k}\left(A, r_{0}\right)$. Dessa forma, dado $\beta_{1} v_{1}=b$ e $x_{k}=V_{k} y_{k}$, a expressão $\left\|b-A x_{k}\right\|_{2}$ pode ser reescrita como:

$$
\left\|b-A x_{k}\right\|_{2}=\left\|\beta_{1} v_{1}-A V_{k} y_{k}\right\|_{2}=\left\|V_{k+1}\left(\beta_{1} e_{1}-H_{k+1, k} y_{k}\right)\right\|_{2} .
$$

Por construção, os vetores colunas da matriz $V_{k+1}$ são ortonormais. Portanto, $V_{k+1}$ é uma transformação ortogonal com respeito ao subespaço de Krylov $\mathcal{K}^{k+1}\left(A, r_{0}\right)$, então:

$$
\left\|b-A x_{k}\right\|_{2}=\left\|\beta_{1} e_{1}-H_{k+1, k} y_{k}\right\|_{2} .
$$

Quando $A$ é simétrica a matriz $H_{k+1, k}$ reduz a uma matriz tridiagonal $T_{k+1, k}$.

O método MINRES é construído utilizando o processo de Lanczos. Dentro de cada etapa de Lanczos, resolvemos o subproblema de quadrados mínimos

$$
y_{k}=\arg \min _{y_{k} \in \mathbb{R}^{k}}\left\|\beta_{1} e_{1}-T_{k+1, k} y_{k}\right\|_{2},
$$

aplicando a fatoração $Q R$

$$
Q_{k} T_{k+1, k}=\left[\begin{array}{c}
R_{k} \\
0
\end{array}\right]=\left[\begin{array}{ccccc}
\gamma_{1}^{(1)} & \delta_{2}^{(1)} & \varepsilon_{3}^{(1)} & & \\
& \ddots & \ddots & \ddots & \\
& & \ddots & \ddots & \varepsilon_{k}^{(1)} \\
& & & \ddots & \delta_{k}^{2} \\
& & & & \gamma_{k}^{(2)} \\
& & & 0
\end{array}\right], \quad Q_{k}\left(\beta_{1} e_{1}\right)=\left[\begin{array}{c}
t_{k} \\
\phi_{k}
\end{array}\right],
$$

em que $Q_{k}=Q_{k, k+1} \cdots Q_{2,3} Q_{1,2}$ é um produto de matrizes ortogonais, construídas para eliminar $\beta_{k+1}$ da subdiagonal de $T_{k+1, k}$ e $t_{k}=\left[\begin{array}{llll}\tau_{1} & \tau_{2} & \cdots & \tau_{k}\end{array}\right]^{t}$. Assim, a solução de quadrados 
mínimos de (3.3) obtida via fatoração QR é definida pelo seguinte sistema triangular: $R_{k} y_{k}=t_{k}$, em que $\left\|r_{k}\right\|_{2}=\left\|b-A x_{k}\right\|_{2}=\left\|\phi_{k}\right\|_{2}^{2}$. Cada $Q_{i, i+1}$ de $Q_{k}$ é definida em termos de $c_{i}$ e $s_{i}$, isto é,

$$
Q_{i, i+1}=\left[\begin{array}{cccc}
I_{i-1} & & & \\
& c_{i} & s_{i} & \\
& s_{i} & -c_{i} & \\
& & & I_{k-i}
\end{array}\right] .
$$

A partir das Rotações de Givens [4, Seção 5.1.8] podemos construir a matriz $Q_{k}$, tal que $Q_{k} T_{k+1, k}$ seja uma matriz triangular superior. Como a matriz $T_{k+1, k}$ é tridiagonal é preciso eliminar a linha abaixo da diagonal principal, assim dados $t_{i i}$ e $t_{i+1, i}$, elementos da matriz $T_{k+1, k}$, obtemos os fatores $c_{i}$ e $s_{i}$ : (Givens $\left(t_{i i}, t i+1, i\right) \rightarrow\left(c_{i}, s_{i}, t_{i i}\right)$ ). O MINRES determina a solução aproximada $x_{k} \in \mathcal{K}^{k}(A, b)$ do problema original $A x=b$ da seguinte forma: $x_{k}=V_{k} y_{k}$. Pela relação $R_{k} y_{k}=t_{k}$ temos que $y_{k}=R_{k}^{-1} t_{k}$. Logo,

$$
\begin{aligned}
x_{k} & =V_{k} y_{k}=V_{k} R_{k}^{-1} t_{k} \\
& =D_{k}\left[\begin{array}{c}
t_{k-1} \\
\tau_{k}
\end{array}\right]=\left[\begin{array}{ll}
D_{k-1} & d_{k}
\end{array}\right]\left[\begin{array}{c}
t_{k-1} \\
\tau_{k}
\end{array}\right] \\
& =x_{k-1}+\tau_{k} d_{k},
\end{aligned}
$$

em que $V_{k} R_{k}^{-1}=D_{k}$, ou melhor, $V_{k}=D_{k} R_{k} \mathrm{e}$

$$
d_{k}=\frac{v_{k}-\delta_{k}^{(2)} d_{k-1}-\varepsilon_{k}^{(1)} d_{k-2}}{\gamma_{k}^{(2)}} .
$$

\section{IMPLEMENTAÇÕES}

Considere o sistema de equações lineares simétrico $A x=b$ e um pré-condicionador para este sistema $M=L^{t} L$. Então o sistema pré-condicionado é equivalente a ao sistema $\left(L^{-1} A L^{-t}\right)\left(L^{t} x\right)=L^{-1} b$, em que $L^{-1} A L^{-t}$ é uma matriz simétrica e definida positiva. Dado o sistema linear $A x=b$, obtemos MINRESP (MINRES pré-condicionado) aplicando MINRES ao sistema equivalente $\hat{A} \hat{x}=\hat{b}$, em que $\hat{A}=L^{-1} A L^{-1}, \hat{x}=L^{-1} x$ e $\hat{b}=L^{-1} b$. Seja $\hat{x} \in \mathcal{K}(\hat{A}, \hat{b})$, $\hat{v}_{0}=0$ e $\hat{\beta}_{1} \hat{v}_{1}=\hat{b}$. Definimos $\hat{z}_{k}=\hat{\beta}_{k} L \hat{v}_{k}$ e $\hat{q}_{k}=\hat{\beta}_{k} L^{-1} \hat{v}_{k}$ tal que $M \hat{q}_{k}=\hat{z}_{k}$. Então,

$$
\hat{\beta}_{k}=\left\|\hat{\beta}_{k} \hat{v}_{k}\right\|_{2}=\left\|L^{-1} \hat{z}_{k}\right\|_{2}=\left(\left(\hat{z}_{k}^{t}\left(L^{-1}\right)^{t}\right)\left(L^{-1} \hat{z}_{k}\right)\right)^{\frac{1}{2}} .
$$

Visto que $M^{-1}=\left(L^{t}\right)^{-1} L^{-1}$ e $M \hat{q}_{k}=\hat{z}_{k}$ temos $\hat{\beta}_{k}=\left(\hat{z}_{k}^{t} M^{-1} \hat{z}_{k}\right)^{\frac{1}{2}}=\sqrt{\hat{q}_{k}^{t} \hat{z}_{k}}$. Dessa forma, obtemos o processo de Lanczos pré-condicionado:

$$
\begin{gathered}
\hat{p}_{k}=\hat{A} \hat{v}_{k}=\frac{1}{\hat{\beta}} L^{-1} A \hat{q}_{k}, \quad \hat{\alpha}_{k}=\hat{v}_{k}^{t} \hat{p}_{k}=\frac{1}{\hat{\beta}^{2}} q_{k}^{t} A \hat{q}_{k} \quad \mathrm{e} \\
\hat{\beta}_{k+1} \hat{v}_{k+1}=L^{-1} A L^{-1} \hat{v}_{k}-\hat{\alpha}_{k} \hat{v}_{k}-\hat{\beta}_{k} \hat{v}_{k-1} .
\end{gathered}
$$


Temos que $q_{k}$ é a solução do sistema $M \hat{q}_{k}=\hat{z}_{k}$. Assim, multiplicando a equação $\hat{\beta}_{k+1} \hat{v}_{k+1}$ por $L$, obtemos:

$$
\hat{z}_{k+1}=\hat{\beta}_{k+1} L \hat{v}_{k+1}=\frac{1}{\hat{\beta}_{k}} A \hat{q}_{k}-\frac{\hat{\alpha}_{k}}{\hat{\beta}_{k}} \hat{z}_{k}-\frac{\hat{\beta}_{k}}{\hat{\beta}_{k-1}} \hat{z}_{k-1} .
$$

Dados o pré-condicionador $M$ e maxit (número máximo de iterações), obtemos a partir do Algoritmo 1 uma solução aproximada $x$ para o sistema $A x=b$.

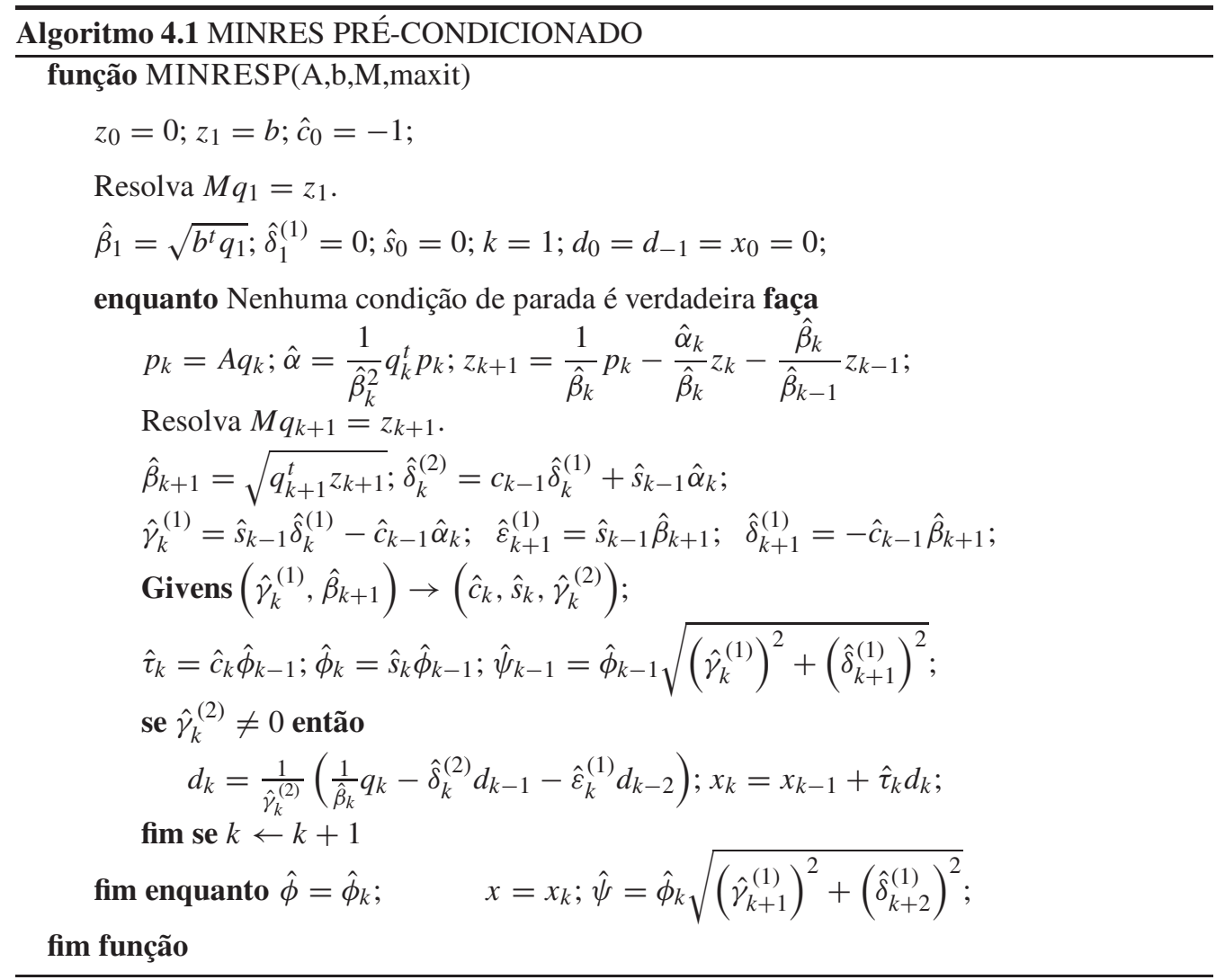

O método MINRESP é construído utilizando o processo de Lanczos pré-condicionado. A cada etapa de Lanczos pré-condicionado, resolvemos o subproblema de quadrados míni-mos equivalente ao apresentado em (3.3) associado ao sistema $\hat{A} \hat{x}=\hat{b}$. Para atualizar a solução do problema original basta multiplicar as equações equivalentes à (3.4) e (3.5), resultadas do MINRES aplicado ao sistema pré-condicionado $\hat{A} \hat{x}=\hat{b}$, por $L^{-1}$ :

$$
d_{k}=L^{-1} \hat{d}_{k}=\frac{1}{\hat{\gamma}_{k}^{(2)}}\left(\frac{1}{\hat{\beta}_{k}} q_{k}-\hat{\delta}_{k}^{(2)} d_{k-1}-\hat{\varepsilon}_{k}^{(1)} d_{k-2}\right) \quad \text { e } \quad x_{k}=L^{-1} \hat{x}_{k}=x_{k-1}+\hat{\tau}_{k} d_{k} .
$$

Um pré-condicionador adequado para GC deve ser simétrico e definido positivo. A ideia do método do GC pré-condicionado (GCP) consiste em aplicar o método GC, como apresentado na Seção 3.3, para resolver um sistema pré-condicionado. Considere o sistema simétrico e definido 
positivo $A x=b$. Pré-condicionando esse sistema obtemos o sistema equivalente: $\hat{A} \hat{x}=\hat{b}$, em que $L$ é uma matriz simétrica e definida positiva. Definimos o pré-condicionador simétrico e definido positivo $M$ por: $M=L^{t} L$. Seja $r_{k}=b-A x_{k}$ o resíduo do sistema original e $z_{k} \mathrm{o}$ resíduo do sistema pré-condicionado tal que $M z_{k}=r_{k}$. Obtemos o processo iterativo do GCP aplicando o GC para resolver o sistema equivalente, isto é,

$$
\begin{aligned}
\alpha_{k} & =\frac{\hat{r}_{k-1}^{t} \hat{r}_{k-1}}{\hat{q}_{k}^{t} \hat{A} \hat{q}_{k}} ; \hat{x}_{k}=\hat{x}_{k-1}+\alpha_{k} \hat{q}_{k} ; \hat{r}_{k}=\hat{r}_{k-1}-\alpha_{k} \hat{A} \hat{q}_{k} ; \\
\lambda_{k+1} & =\frac{\hat{r}_{k}^{t} \hat{r}_{k}}{\hat{r}_{k-1}^{t} \hat{r}_{k-1}} ; \hat{q}_{k+1}=\hat{r}_{k}+\lambda_{k+1} \hat{q}_{k} .
\end{aligned}
$$

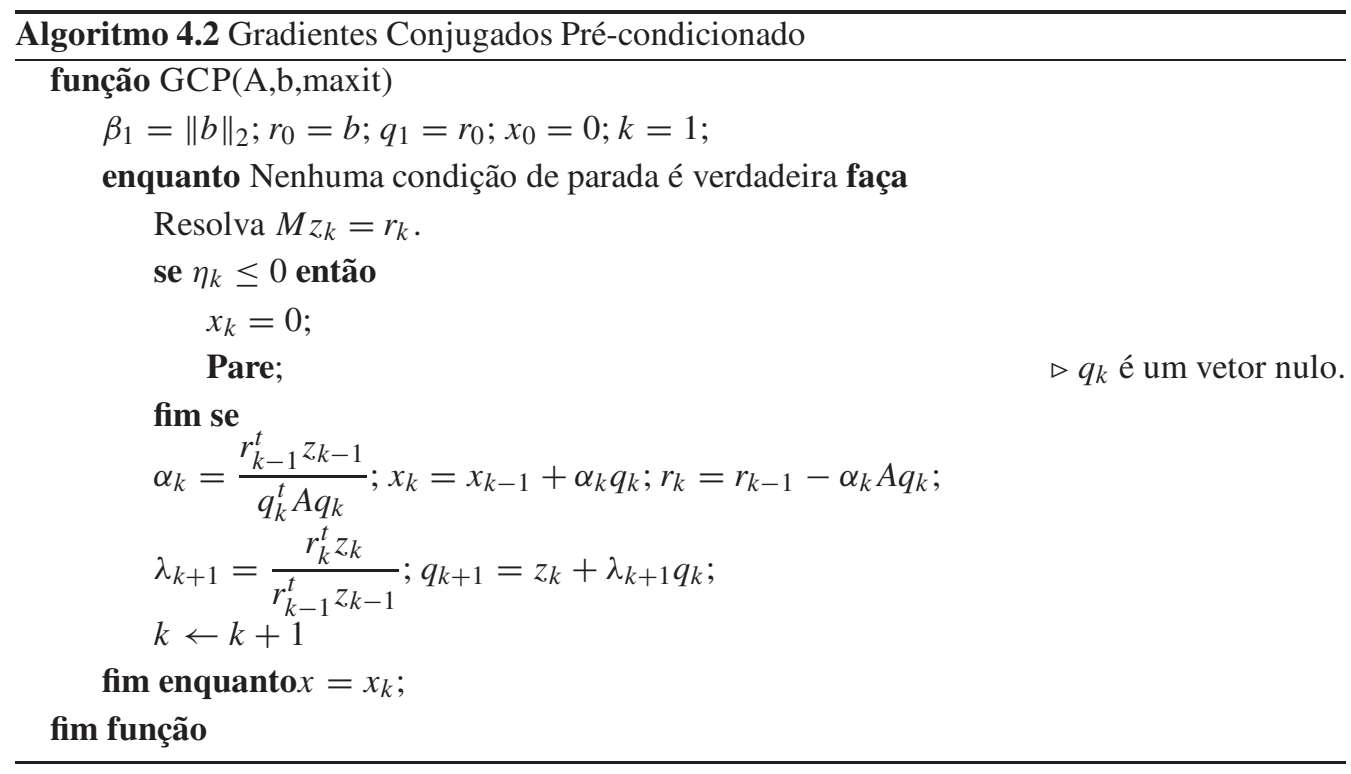

Visto que a matriz $A D A^{t}$ do sistema de equações normais (2.5) é simétrica e definida positiva, podemos utilizar o GCP para determinar as direções de Newton nos métodos de pontos interiores. Esta versão do método do GCP fornece uma solução aproximada do sistema original diretamente sem ter que calculá-la a partir da solução aproximada do sistema de pré-condicionado. Os resultados teóricos e experimentais apresentados em [2] fornecem evidências que muitas vezes, em sistemas simétricos e definido positivo, o MINRES pode parar muito mais cedo que o GC. Em alguns casos, o MINRES pode convergir mais rapidamente que o GC. Porém, geralmente o GC converge mais rapidamente do que o MINRES considerando tanto $\left\|x^{*}-x_{k}\right\|_{A}{ }^{6}$ quanto $\left\|x^{*}-x_{k}\right\|_{2}$. Nos trabalhos [13] e [5], são feitas comparações entre os métodos MINRES, CG e também outros métodos de resolução de sistemas. A seguir, apresentamos o Algoritmo 3 para a nova abordagem proposta, a qual utiliza os métodos GCP e MINRESP juntos.

\footnotetext{
${ }^{6}$ A norma-A é definida como:
}

$$
\|w\|_{A}=\sqrt{w^{t} A w}
$$




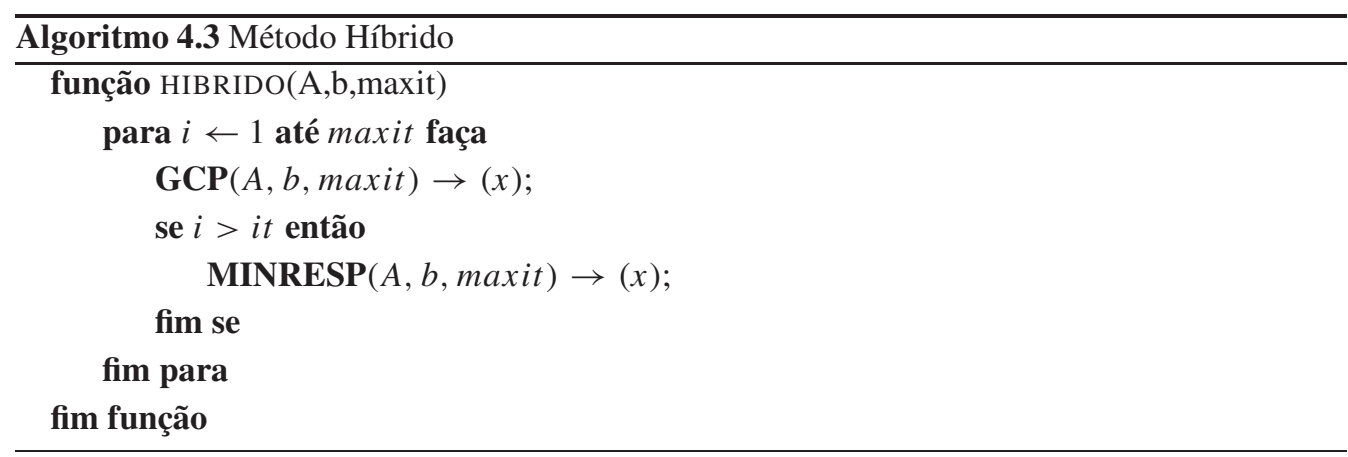

Para resolver um sistema $A x=b$, o Algoritmo 3 utiliza, inicialmente, o GCP. Se este método não convergir até um certo número de iterações (it) pré-determinado, então o MINRESP passa a ser utilizado para resolver o sistema. Nos experimentos, consideramos $i t=$ número de linhas da matriz dos coeficientes.

\section{EXPERIMENTOS COMPUTACIONAIS}

Para comparar o desempenho do método preditor-corretor ao resolver problemas de programação linear usando os métodos iterativos GC e MINRES, ambos pré-condicionados pelo précondicionador separador e uma nova abordagem híbrida, a qual utiliza, inicialmente, o CGP e em seguida o MINRESP, foram realizados experimentos computacionais em um Intel Core i5, 4 GB RAM, 2,3 GHZ, 225 GB HD, com sistema operacional Linux. O código foi implementado em linguagem C e os 63 problemas de programação linear testados, pertencentes as coleções Netlib [3], Qaplib [1], Kennington [7], STOCHLP [17] e MISC [11], que estão no formato MPS, foram lidos usado callable library do CPLEX.

O pré-condicionador separador requer o cálculo da fatoração LU da matriz do sistema. Uma nova fatoração LU é calculada de uma iteração para a outra sempre que os métodos GCP e MINRESP necessitam muitas iterações para atingir a convergência. Em [14] é proposto calcular uma segunda fatoração LU(refatoração) quando a primeira fatoração é muito densa a fim de melhorar o desempenho. Esta técnica foi considerada nos experimentos. As Tabelas 1 e 2 comparam o tempo total e número de iterações, respectivamente, do método preditor-corretor ao resolver os problemas de programação linear usando os métodos ite-rativos GCP e MINRESP. Foram resolvidos 38 problemas pelo GCP e/ou MINRESP. Nas colunas Refact. a refatoração está sendo realizada.

Os primeiros experimentos foram feitos considerando apenas o sistema aumentado e depois o sistema de equações normais. No primeiro caso, o método GCP não convergiu para nenhum problema enquanto o MINRESP mostrou-se mais robusto e apresentou bons resultados resolvendo 25 problemas, sendo que 2 deles foram resolvidos somente nesse caso. Considerando o sistema de equações normais os dois métodos apresentaram resultados satisfatórios e equilibrados. Quando a refatoração é feita, o GCP resolveu 3 problemas a mais que o MINRESP e em menos tempo em $50 \%$ dos problemas, porém a diferença no tempo entre os métodos não 
Tabela 1: Tempo total do método de pontos interiores em segundos.

\begin{tabular}{|c|c|c|c|c|c|c|c|c|}
\hline \multirow{3}{*}{ Problema } & \multicolumn{4}{|c|}{ Sistema Aumentado } & \multicolumn{4}{|c|}{ Equações Normais } \\
\hline & \multicolumn{2}{|c|}{ Refact. } & \multicolumn{2}{|c|}{ Fact. } & \multicolumn{2}{|c|}{ Refact. } & \multicolumn{2}{|c|}{ Fact. } \\
\hline & GCP & MINRESP & GCP & MINRESP & GCP & MINRESP & GCP & MINRESP \\
\hline afiro & $*$ & 0.00 & $*$ & 0.00 & 0.00 & 0.00 & 0.00 & 0.00 \\
\hline agg & $*$ & $*$ & $*$ & $*$ & $*$ & $*$ & 1.20 & $*$ \\
\hline fit1d & $*$ & 0.49 & $*$ & 0.36 & $*$ & 0.28 & $*$ & 0.06 \\
\hline fit $2 d$ & $*$ & 14.78 & $*$ & $*$ & $*$ & 15.49 & $*$ & 2.48 \\
\hline ganges & * & $*$ & $*$ & $*$ & 1.52 & $*$ & $*$ & $*$ \\
\hline gen4 & $*$ & $*$ & $*$ & 16.32 & $*$ & $*$ & $*$ & $*$ \\
\hline $\mathrm{kb} 2$ & * & 0.04 & $*$ & * & 0.02 & $*$ & 0.01 & $*$ \\
\hline ken-07 & * & 0.86 & $*$ & 0.88 & 0.61 & 0.75 & 0.60 & 0.27 \\
\hline nug05 & $*$ & $*$ & $*$ & 0.1 & 0.08 & 0.09 & 0.70 & 0.03 \\
\hline nug06 & $*$ & $*$ & $*$ & * & 0.42 & 0.52 & $*$ & $*$ \\
\hline nug07 & * & $*$ & $*$ & * & 4.66 & $*$ & 5.19 & 4.66 \\
\hline nug30 & * & $*$ & $*$ & $*$ & $*$ & 5.57 & $*$ & $*$ \\
\hline pds-02 & $*$ & 7.8 & $*$ & 7.82 & 4.68 & $*$ & 4.69 & 2.1 \\
\hline qap8 & * & $*$ & $*$ & * & * & $*$ & 18.73 & $*$ \\
\hline recipe & $*$ & 0.03 & $*$ & 0.03 & 0.02 & 0.02 & 0.02 & 0.00 \\
\hline $\operatorname{sc50a}$ & * & 0.01 & $*$ & 0.00 & 0.00 & 0.00 & 0.00 & 0.00 \\
\hline $\mathrm{sc} 50 \mathrm{~b}$ & $*$ & 0.00 & $*$ & 0.00 & 0.00 & 0.00 & 0.00 & 0.00 \\
\hline $\mathrm{sc} 105$ & $*$ & 0.03 & $*$ & 0.01 & 0.01 & 0.00 & 0.01 & 0.01 \\
\hline sc205 & $*$ & $*$ & $*$ & $*$ & $*$ & $*$ & 0.21 & $*$ \\
\hline scagr7 & * & 0.03 & $*$ & 0.02 & 0.01 & 0.01 & 0.01 & 0.01 \\
\hline scsd1 & * & 0.10 & $*$ & 0.08 & $*$ & 0.06 & $*$ & 0.02 \\
\hline scsd6 & $*$ & 0.24 & $*$ & 0.23 & 0.14 & 0.16 & 0.14 & 0.05 \\
\hline $\operatorname{scsd} 8$ & $*$ & $*$ & $*$ & $*$ & 0.54 & $*$ & 0.52 & $*$ \\
\hline sctap1 & $*$ & 0.33 & $*$ & 0.32 & 0.25 & 0.26 & 0.23 & 0.08 \\
\hline sctap2 & $*$ & 1.14 & * & 1.14 & 2.25 & 0.89 & 2.25 & 0.29 \\
\hline sctap3 & $*$ & 21.72 & $*$ & $*$ & 66.2 & $*$ & $*$ & $*$ \\
\hline share $2 b$ & * & 0.31 & * & 0.21 & 0.11 & 0.12 & * & $*$ \\
\hline shell & $*$ & 3.12 & $*$ & 3.18 & 0.57 & 0.94 & 0.53 & $*$ \\
\hline ship041 & $*$ & 0.36 & $*$ & 0.31 & 0.24 & 0.27 & 0.18 & 0.08 \\
\hline ship04s & $*$ & $*$ & $*$ & * & * & $*$ & $*$ & 0.21 \\
\hline ship081 & $*$ & * & $*$ & * & * & $*$ & $*$ & 0.37 \\
\hline ship08s & $*$ & 0.36 & $*$ & 0.34 & 0.25 & 0.28 & 0.22 & 0.08 \\
\hline ship121 & $*$ & 1.17 & $*$ & 1.22 & 0.8 & 0.87 & 0.71 & 0.26 \\
\hline ship12s & $*$ & 0.48 & $*$ & 0.46 & 0.33 & 0.37 & 0.3 & 0.12 \\
\hline standgub & $*$ & 1.35 & $*$ & 1.44 & 0.59 & 1.57 & 0.57 & 0.24 \\
\hline standmps & * & 1.93 & * & 2.52 & 0.79 & 1.7 & 1.40 & 0.48 \\
\hline stocfor1 & $*$ & 0.03 & $*$ & 0.05 & 0.04 & 0.36 & 0.05 & $*$ \\
\hline truss & $*$ & $*$ & $*$ & * & 12.45 & $*$ & $*$ & $*$ \\
\hline
\end{tabular}


Tabela 2: Total de iterações do método de pontos interiores.

\begin{tabular}{|c|c|c|c|c|c|c|c|c|}
\hline \multirow{3}{*}{ Problema } & \multicolumn{4}{|c|}{ Sistema Aumentado } & \multicolumn{4}{|c|}{ Equações Normais } \\
\hline & \multicolumn{2}{|c|}{ Refact. } & \multicolumn{2}{|c|}{ Fact. } & \multicolumn{2}{|c|}{ Refact. } & \multicolumn{2}{|c|}{ Fact. } \\
\hline & GCP & MINRESP & GCP & MINRESP & $\overline{\mathrm{GCP}}$ & MINRESP & GCP & MINRESP \\
\hline agg & $*$ & $*$ & * & $*$ & $*$ & $*$ & 36 & $*$ \\
\hline fit1d & $*$ & 31 & $*$ & 26 & $*$ & 24 & * & 24 \\
\hline fit2d & * & 95 & $*$ & $*$ & $*$ & 121 & $*$ & 88 \\
\hline ganges & * & $*$ & $*$ & $*$ & 20 & $*$ & $*$ & $*$ \\
\hline gen4 & * & $*$ & $*$ & 43 & * & $*$ & * & * \\
\hline $\mathrm{kb} 2$ & $*$ & 22 & $*$ & $*$ & 11 & $*$ & 11 & * \\
\hline nug05 & $*$ & $*$ & $*$ & 8 & 8 & 8 & 8 & 8 \\
\hline nug06 & $*$ & $*$ & $*$ & $*$ & 9 & 9 & * & $*$ \\
\hline nug07 & * & $*$ & $*$ & $*$ & 14 & $*$ & 12 & 14 \\
\hline nug30 & $*$ & $*$ & $*$ & $*$ & * & 29 & $*$ & $*$ \\
\hline pds-02 & $*$ & 29 & $*$ & 29 & 29 & $*$ & 29 & 29 \\
\hline qap8 & $*$ & $*$ & $*$ & $*$ & $*$ & $*$ & 10 & $*$ \\
\hline $\operatorname{sc} 205$ & $*$ & $*$ & $*$ & $*$ & * & $*$ & 18 & $*$ \\
\hline scagr7 & $*$ & 16 & $*$ & 16 & 10 & 16 & 16 & 16 \\
\hline $\operatorname{scsd} 1$ & $*$ & 11 & $*$ & 11 & $*$ & 11 & $*$ & 11 \\
\hline $\operatorname{scsd} 8$ & $*$ & $*$ & $*$ & $*$ & 11 & $*$ & 11 & $*$ \\
\hline sctap2 & $*$ & 17 & $*$ & 17 & 24 & 17 & 24 & 17 \\
\hline sctap3 & $*$ & 32 & $*$ & $*$ & 91 & $*$ & * & $*$ \\
\hline share $2 b$ & $*$ & 29 & $*$ & 19 & 19 & 19 & $*$ & $*$ \\
\hline shell & $*$ & 36 & $*$ & 26 & 23 & 24 & 23 & $*$ \\
\hline ship04s & $*$ & $*$ & $*$ & $*$ & $*$ & $*$ & $*$ & 20 \\
\hline ship081 & $*$ & $*$ & $*$ & $*$ & $*$ & $*$ & $*$ & 16 \\
\hline standgub & * & 25 & $*$ & 24 & 22 & 23 & 22 & 22 \\
\hline standmps & $*$ & 28 & $*$ & 30 & 30 & 29 & 32 & 28 \\
\hline stocfor1 & $*$ & 13 & * & 17 & 15 & 54 & 16 & $*$ \\
\hline truss & $*$ & $*$ & $*$ & $*$ & 18 & $*$ & $*$ & $*$ \\
\hline
\end{tabular}

foi muito significante. Com relação ao número de iterações o GCP fez menos iterações em 9 problemas contra 6 problemas do MINRESP.

Considerando o caso em que a refatoração não é feita, observa-se um melhor desempenho do MINRESP, que foi mais rápido em todos os problemas resolvidos por ambos métodos. A diferença nos tempos foi bem significante, chegando a ser 23 vezes menor em um dos problemas. Com relação ao número de iterações os resultados foram muito próximos.

Na Tabela 2, foram apresentados os resultados somente dos problemas em que houveram diferenças no número total de iterações. Com os experimentos já realizados, observamos que aproximadamente $34 \%$ dos problemas testados foram resolvidos somente por um dos métodos MINRESP ou GCP. Isso motivou a realização de mais um teste considerando o Algoritmo 3. 
De acordo com os resultados obtidos, temos que o método MINRESP sem refatorar e usando o sistema de equações normais apresentou o melhor desempenho com relação ao tempo e como o GCP não funciona bem com o sistema aumentado, decidimos utililizar o método híbrido com sistema de equações normais e sem refatoração. A Tabela 3 compara os resultados desse novo teste.

Tabela 3: Comparação entre GCP, MINRESP e Híbrido.

\begin{tabular}{|c|c|c|c|c|c|c|}
\hline \multirow{2}{*}{ Problema } & \multicolumn{2}{|l|}{ GCP } & \multicolumn{2}{|c|}{ MINRESP } & \multicolumn{2}{|c|}{ Híbrido } \\
\hline & Tempo(s) & It. & Tempo(s) & It. & Tempo(s) & It. \\
\hline adlittle & $*$ & $*$ & $*$ & $*$ & 0.01 & 13 \\
\hline afiro & 0.00 & 9 & 0.00 & 9 & 0.00 & 9 \\
\hline agg & 1.20 & 36 & $*$ & $*$ & 0.39 & 23 \\
\hline agg3 & $*$ & $*$ & * & * & 2.17 & 25 \\
\hline bandm & $*$ & $*$ & $*$ & $*$ & 2.90 & 47 \\
\hline beaconfd & $*$ & $*$ & $*$ & $*$ & 1.93 & 64 \\
\hline chr22b & $*$ & $*$ & $*$ & $*$ & 30.64 & 30 \\
\hline chr25a & $*$ & $*$ & $*$ & $*$ & 60.26 & 31 \\
\hline cre-a & $*$ & $*$ & $*$ & $*$ & 28.48 & 33 \\
\hline czprob & $*$ & $*$ & $*$ & $*$ & 0.59 & 33 \\
\hline els19 & $*$ & $*$ & $*$ & $*$ & 810.02 & 31 \\
\hline finnis & $*$ & $*$ & $*$ & $*$ & 1.21 & 26 \\
\hline fit1d & $*$ & $*$ & 0.06 & 24 & 0.29 & 25 \\
\hline fit1p & $*$ & $*$ & $*$ & $*$ & 1.11 & 23 \\
\hline fit $2 d$ & $*$ & $*$ & 2.48 & 88 & 11.18 & 57 \\
\hline ganges & $*$ & $*$ & $*$ & $*$ & 1.37 & 20 \\
\hline israel & $*$ & $*$ & $*$ & $*$ & 0.48 & 22 \\
\hline $\mathrm{kb} 2$ & 0.01 & 11 & $*$ & $*$ & 0 & 11 \\
\hline ken 11 & $*$ & $*$ & $*$ & $*$ & 15.91 & 23 \\
\hline ken13 & $*$ & $*$ & $*$ & $*$ & 68.33 & 25 \\
\hline ken-07 & 0.60 & 16 & 0.27 & 16 & 0.54 & 16 \\
\hline lotfi & $*$ & $*$ & $*$ & $*$ & 3.17 & 101 \\
\hline nug05 & 0.70 & 8 & 0.03 & 8 & 0.07 & 8 \\
\hline nug06 & $*$ & $*$ & $*$ & $*$ & 0.43 & 9 \\
\hline nug07 & 5.19 & 12 & 4.66 & 14 & 2.72 & 12 \\
\hline nug08 & $*$ & $*$ & $*$ & $*$ & 10.45 & 10 \\
\hline pds-02 & 4.69 & 29 & 2.10 & 29 & 4.06 & 29 \\
\hline pds-06 & $*$ & $*$ & $*$ & $*$ & 57.87 & 38 \\
\hline pds-10 & $*$ & $*$ & * & $*$ & 188.27 & 54 \\
\hline qap8 & 18.73 & 10 & $*$ & $*$ & 9.79 & 10 \\
\hline recipe & 0.02 & 11 & 0.00 & 11 & 0.03 & 11 \\
\hline
\end{tabular}


Tabela 3: (continuação)

\begin{tabular}{|c|c|c|c|c|c|c|}
\hline \multirow{2}{*}{ Problema } & \multicolumn{2}{|c|}{ GCP } & \multicolumn{2}{c|}{ MINRESP } & \multicolumn{2}{c|}{ Híbrido } \\
\cline { 2 - 7 } & Tempo(s) & It. & Tempo(s) & It. & Tempo(s) & It. \\
\hline sc50a & 0.00 & 10 & 0.00 & 10 & 0.00 & 10 \\
\hline sc50b & 0.00 & 9 & 0.00 & 9 & 0.00 & 9 \\
\hline sc105 & 0.01 & 10 & 0.01 & 10 & 0.01 & 10 \\
\hline sc205 & 0.21 & 18 & $*$ & $*$ & 0.04 & 11 \\
\hline scagr7 & 0.01 & 10 & 0.01 & 16 & 0.01 & 16 \\
\hline scr15 & $*$ & $*$ & $*$ & $*$ & 72.19 & 23 \\
\hline scr20 & $*$ & $*$ & $*$ & $*$ & 1219.38 & 24 \\
\hline scsd1 & $*$ & $*$ & 0.02 & 11 & 0.07 & 11 \\
\hline scsd6 & 0.14 & 11 & 0.05 & 11 & 0.15 & 11 \\
\hline scsd8 & 0.52 & 11 & $*$ & $*$ & 0.54 & 11 \\
\hline scsd8-2b-64 & $*$ & $*$ & $*$ & $*$ & 17.83 & 12 \\
\hline scsd8-2c-64 & $*$ & $*$ & $*$ & $*$ & 20.77 & 11 \\
\hline scsd8-2r-432 & $*$ & $*$ & $*$ & $*$ & 48.28 & 18 \\
\hline sctap1 & 0.23 & 19 & 0.08 & 19 & 0.2 & 19 \\
\hline sctap2 & 2.25 & 24 & 0.29 & 17 & 0.69 & 17 \\
\hline sctap3 & $*$ & $*$ & $*$ & $*$ & 1.24 & 20 \\
\hline share1b & $*$ & $*$ & $*$ & $*$ & 0.15 & 26 \\
\hline share2b & $*$ & $*$ & $*$ & $*$ & 0.05 & 12 \\
\hline shell & 0.53 & 23 & $*$ & $*$ & 0.29 & 23 \\
\hline ship041 & 0.18 & 15 & 0.08 & 15 & 0.23 & 15 \\
\hline ship04s & $*$ & $*$ & 0.21 & 20 & 0.14 & 16 \\
\hline ship081 & $*$ & $*$ & 0.37 & 16 & 0.6 & 18 \\
\hline ship08s & 0.22 & 16 & 0.08 & 16 & 0.24 & 16 \\
\hline ship121 & 0.71 & 18 & 0.26 & 18 & 0.76 & 18 \\
\hline ship12s & 0.30 & 17 & 0.12 & 17 & 0.32 & 17 \\
\hline standgub & 0.57 & 22 & 0.24 & 22 & 0.54 & 22 \\
\hline standmps & 1.4000 & 32 & 0.48 & 28 & 0.73 & 30 \\
\hline stocfor1 & 0.0500 & 16 & $*$ & $*$ & 0.02 & 13 \\
\hline stocfor2 & $*$ & $*$ & $*$ & $*$ & 7.91 & 32 \\
\hline truss & $*$ & $*$ & $*$ & $*$ & 11.52 & 18 \\
\hline & & & & & \\
\hline
\end{tabular}

O método híbrido resolveu 61 dos 63 problemas testados, o que significa que 25 problemas passaram a ser solucionados com a nova abordagem. Além disso, este método foi mais rápido em $62 \%$ dos problemas, o MINRESP em $27 \%$ e o GCP em aproximadamente $2 \%$ dos problemas. O número de iterações foi menor em $59 \%$ dos problemas para o híbrido, $6 \%$ para o MINRESP e em torno de $2 \%$ para o GCP. Considerando os problemas resolvidos apenas pelo GCP e híbrido, 
vemos que somente um deles foi resolvido em menor tempo pelo GCP. Com relação ao número de iterações, o valor foi menor ou igual para o híbrido em todos os problemas. Comparando os resultados do MINRESP com o híbrido vemos que somente 2 problemas foram resolvidos mais rápido pelo híbrido e apenas 3 problemas tiveram o número de iterações menor.

\title{
6 CONCLUSÕES
}

Neste trabalho, para a resolução dos sistemas lineares a cada iteração do método preditor-corretor consideramos tanto o sistema aumentado quanto o sistema de equações normais. O método MINRESP com reortogonalização foi implementado e comparado com o método dos gradientes conjugados, ambos métodos foram pré-condicionados pelo pré-condiciona-dor separador. Experimentos computacionais foram realizados com o objetivo de determinar qual método é mais eficiente e robusto. Os resultados mostraram que o MINRESP é mais robusto que o GCP quando somente o sistema aumentado é considerado. Para o sistema de equações normais com e sem refatoração os resultados foram semelhantes para o número de iterações, porém o tempo foi menor ou igual em todos os problemas para o MINRESP sem refatorar. Um método híbrido também foi considerado, o qual resolveu $40 \%$ a mais de problemas (inclusive problemas de dimensões maiores) além de ser mais rápido e fazer menos iterações que os métodos GCP e MINRESP para o sistema de equações normais sem refatoração. Dessa forma, concluímos que este método é o mais robusto e eficiente e será ainda mais investigado e utilizado em trabalhos futuros.

\section{AGRADECIMENTOS}

Ao CNPq e a FAPESP pelo apoio financeiro.

\begin{abstract}
In this paper, we consider the predictor-corrector method, which is one of the most important variants of the interior point methods due to its efficiency and fast convergence. In the predictor-corrector method it is necessary to solve two linear systems at each iteration to determine the search direction. The solution of such systems is the step that requires more processing time and should therefore be carried out efficiently. Two iterative Krylov methods are considered to solve these linear systems of: the MINRES and the conjugate gradient method. A preconditioner specially developed for the linear systems arising from the interior point methods is used so that the iterative methods converge faster. Computational experiments in a some sets of linear programming problems are performed in order to analyse the efficiency and robustness of the linear systems solution methods.
\end{abstract}

Keywords: interior point methods, linear systems, iterative methods.

\section{REFERÊNCIAS}

[1] R.S. Burkard, S. Karisch \& F. Rendl. Qaplib - a quadratic assignment problem library. European Journal of Operations Research, 55 (1991), 115-119. 
[2] D.C. Fong \& M. A. Saunders. CG versus MINRES: An empirical comparison. SQU Journal for Science, 17(1) (2012), 44-62.

[3] D.M. Gay. Electronic mail distribution of linear programming test problems. Mathematical Programming Society Committee on Algorithms COAL Newsletter, (1985), 10-12.

[4] G.H. Golub \& C.F. Van Loan. Matrix Computations. $3^{\text {a }}$ ed. Baltimore, Md., London: The Johns Hopkins University Press, (1996).

[5] I.B. Kalambi. A Comparison of three Iterative Methods for the Solution of Linear Equations. Journal of Applied Sciences and Environmental Management, 12(4) (2009), 53-55.

[6] N. Karmarkar. A new polynomial-time algorithm for linear programming. Combinato-rica, 4 (1984), 373-395.

[7] J.L. Kennington, S. Niemi, W.J. Carolan, J.E. Hill \& S.J. Wichmann. An empirical evaluation of the korbx algorithms for military airlift applications. Oper. Res, 38 (1990), 240-248.

[8] C. Lanczos. An iteration method for the solution of the eigenvalue problem of linear differential and integral operators. J. Res. Nat. Bureau Standards, 45(4) (1950), 255-282.

[9] S. Mehrotra. On the implementation of a primal-dual interior point method. SIAM Journal on Optimization, 2(4) (1992), 575-601.

[10] C.D. Meyer. Matrix analysis and applied linear algebra. SIAM Publications, SIAM, (2000).

[11] Miscellaneous lp models. Technical report, Hungarian Academy of Sciences OR Lab. url, www.sztaki.hu/ meszaros/publicftp/lptestset/misc .

[12] R.D.C. Monteiro, I. Adler \& M.G.C. Resende. A polynomial-time primal-dual affine scaling algorithm for linear and convex quadratic programming and its power series extension. Mathematics of Operations Research, 15 (1990), 191-214.

[13] J. Noreen. A comparison of direct and indirect solvers for linear system of equations. International Journal of Emerging Science, 2(2) (2012), 310-321.

[14] A.R.L. Oliveira \& D.C. Sorensen. A new class of preconditioners for large-scale linear systems from interior point methods for linear programming. Linear Algebra and Its Applications, 394 (2005), $1-24$.

[15] Y. Saad. Iterative Methods for Sparse Linear Systems, SIAM, (2003).

[16] D.C. Sorensen. Implicit application of polynomial filters in a $k$-step Arnoldi method. SIAM Journal on Matrix Analysis and Applications, 13 (1992), 357-385.

[17] Stochastic lp test sets. Technical report, Hungarian Academy of Sciences OR Lab. url, www.sztaki.hu/ meszaros/publicftp/lptestset/stochlp.

[18] S.J. Wright. Primal-dual interior-point methods. SIAM Publications, SIAM, Philadelphia, PA, USA, (1997). 